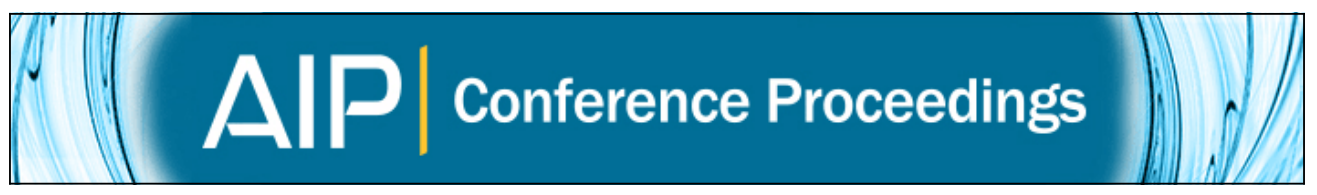

\title{
Dynamical aspects of fragmentation
}

M. J. Ison and C. O. Dorso

Citation: AIP Conference Proceedings 884, 513 (2007); doi: 10.1063/1.2710647

View online: http://dx.doi.org/10.1063/1.2710647

View Table of Contents: http://scitation.aip.org/content/aip/proceeding/aipcp/884?ver=pdfcov

Published by the AIP Publishing

\section{Articles you may be interested in}

Zipf's Law and the Universality Class of the Fragmentation Phase Transition

AIP Conf. Proc. 884, 327 (2007); 10.1063/1.2710600

From multifragmentation to supernovae and neutron stars

AIP Conf. Proc. 884, 318 (2007); 10.1063/1.2710599

Production of complex particles in low energy spollation and in fragmentation reactions by inmedium random clusterization

AIP Conf. Proc. 791, 112 (2005); 10.1063/1.2114699

Isoscaling and symmetry energy in dynamical fragment formation

AIP Conf. Proc. 791, 83 (2005); 10.1063/1.2114696

A non perturbative approach to neutron and proton halo breakup

AIP Conf. Proc. 704, 218 (2004); 10.1063/1.1737114 


\title{
Dynamical aspects of fragmentation
}

\author{
M. J. Ison and C. O. Dorso \\ Departamento de Física, Facultad de Ciencias Exactas y Naturales, Universidad de Buenos Aires, \\ Pabellon I, Ciudad Universitaria, Nuñez, 1428, Buenos Aires, Argentina.
}

Keywords: Nonequilibrium thermodynamics, fragmentation, reducibility

PACS: $25.70 . \mathrm{Mn}, 25.70 \mathrm{-z}, 25.70 . \mathrm{Pq}, 02.70 . \mathrm{Ns}$

Fragmentation of hot nuclear systems has been the subject of several studies during the recent past. Because infinite nuclear matter has an equation of state very similar to that of a Van der Waals gas [1], which exhibits a liquid-gas phase transition, many generic approaches have been developed to provide a general framework for the nuclear problem including statistical models (like the lattice gas model) $[2,3]$ and dynamical ones (including classic dynamical models $[4,5]$ ).

The advantage of working with models which are fully microscopic is that both equilibrium and nonequilibrium features of the problem can be explored.

Several aspects of the multifagmentation process are still a matter of debate. In particular, the problem of sequentiality versus simultaneity $[6,7]$ arose since Moretto and coworkers proposed that the complex behavior of fragment emission could be described in terms of a simple binomial distribution, namely:

$$
P_{n}^{m}=\frac{m !}{n !(m-n) !} p^{n}(1-p)^{m-n}
$$

Where $m$ stands for the number of "trials", and $n$ represent the number of successes. Following [6], we associate the parameter $m$ to the maximum multiplicity for each energy and $p$ to the emission probability. It is interesting that $m$ could possibly be think of as the number of natural time intervals at which the system fragments with probability $p$. This approach rests on the assumption that a single fragmentation probability is capable of describing the emission process disregarding the characteristics of the emitting source.

From the experimental side, it was found that in many reactions the multiplicity of intermediate-mass-fragments (IMF, with charges $Z=3-20$ ) is distributed as a function of the transverse energy $E_{t}$ binomially. This energy is assumed to be proportional to the excitation energy $E$, which is related to the temperature, considering the system as a Fermi gas, via $T \propto \sqrt{E}$, so that $p \propto e^{-B / T}$, an Arrhenius law. Then, it is inferred that multifragmentation is a sequence of thermal binary events.

We performed molecular dynamics calculation of a system composed of $N=147$ particles interacting via a truncated Lennard-Jones potential. Initial configurations were prepared, like in previous works $[4,5]$, as hot dense drops. In order to get close to the experimental situation, no artificial constraining volumes were introduced, i.e. particles

CP884, VI Latin American Symposium on Nuclear Physics and Applications,

edited by O. Civitarese, C. Dorso, G. García Bermúdez, A. J. Kreiner, A. J. Pacheco, and N. N. Scoccola (C) 2007 American Institute of Physics 978-0-7354-0388-8/07/\$23.00 


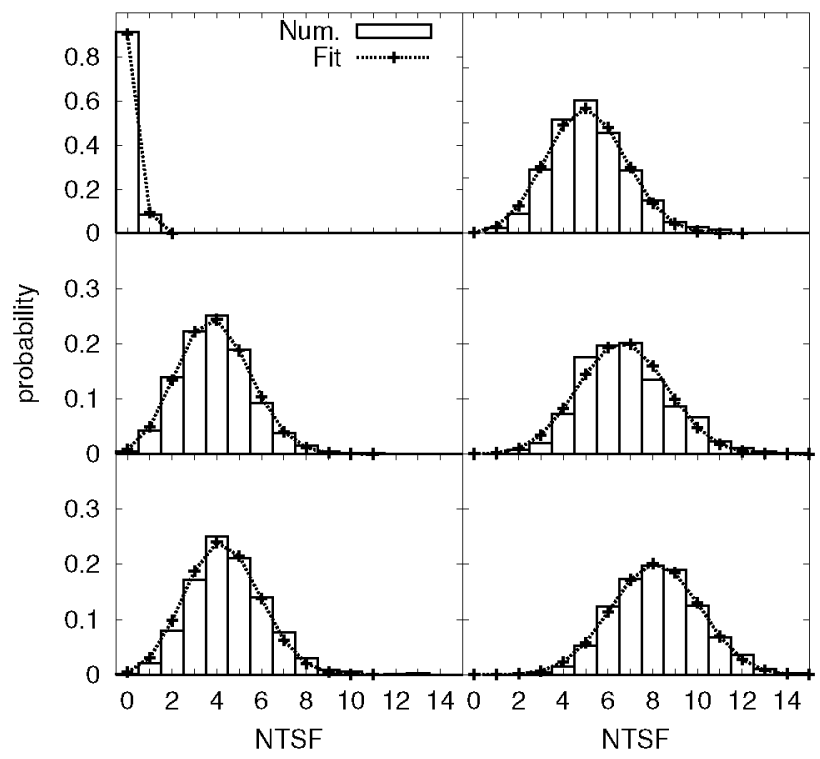

FIGURE 1. Fragmentation probability as a function of the number of times the system fragments (NTSF) (histograms) and binomial fit (dotted line). For energies (from top to bottom and left to right): $E=-2.0 \varepsilon, E=-0.2 \varepsilon, E=0.0 \varepsilon, E=0.2 \varepsilon, E=1.0 \varepsilon$, and $E=2.0 \varepsilon$

were freely evolving into vacuum. We define a fragmentation process when a source emits a quasi-stable fragment of at least four particles. Fragments are detected in configuration space and our temporal stability criterion is that a fragment is considered as quasi-stable when particles remain together for at least $5 t_{0}$ (in natural Lennard-Jones units).

In Figure 1 we show that the distribution of emitted fragments is well adjusted with a binomial distribution. The agreement is remarkable specially when one considers that we are facing an out-of-equilibrium process and exploring a wide energy range, from $E=-2.0 \varepsilon$, which has a U-shaped mass spectra, to $E=2.0 \varepsilon$, characterized by an exponentially decaying mass distribution.

To further analyze the implications of this approach we show in Fig.2 the relationship between the emission probability and the energy. To investigate if this relationship could indeed correspond to an Arrhenius law we plot the inverse of the probability as a function of the inverse of the excitation energy (right panel of Fig.2). Almost all the points line up with a straight line. However if we are to test if the Arrhenius law hold we still need to calculate the temperature, which should have a dependence of the type $T \propto E^{*}$. The temperature of the emitting source was calculated in Ref.[4] and the values of $T$ were approximately linear with $E$ only for low energy values, which suggest that the Arrhenius law could be fulfilled only when evaporation is the principal decaying mode. 


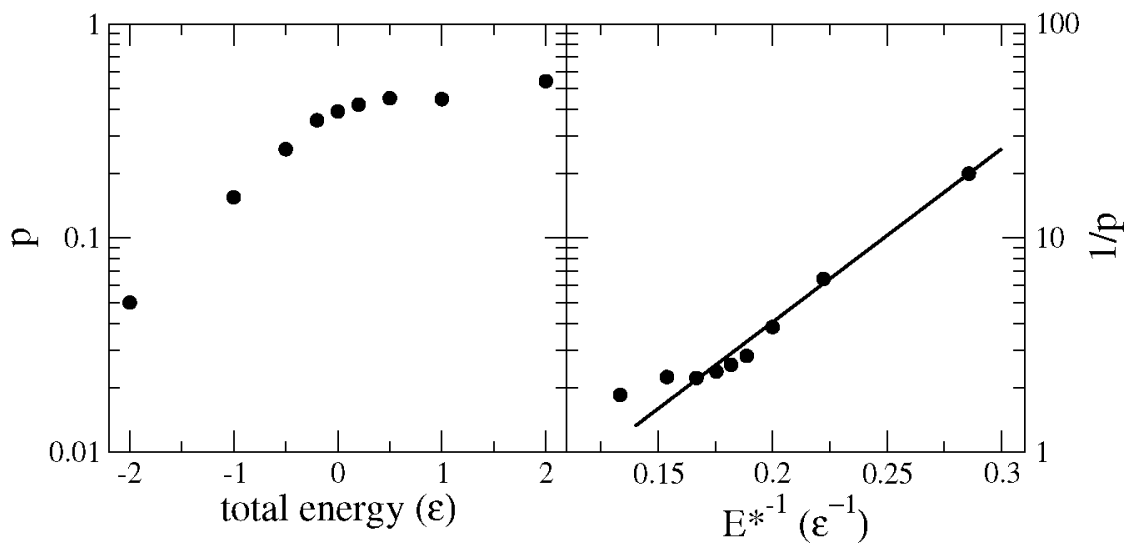

FIGURE 2. Fragmentation probability as a function of total energy (left) and reciprocal of the probability as a function of the inverse of the excitation energy (right)

Moreover, an analysis of the fragmentation times was performed and showed that fragmentation events are more likely to occur at rather early stages of the evolution. The picture that emerges is that this process can be viewed as a mixture of sequential and simultaneous breakups.

\section{REFERENCES}

1. A. Vicentini, G. Jacucci and V. R. Pandharipande, Phys. Rev. C 311783 (1985).

2. C. B. Das, L. Shi and S. Das Gupta, Phys. Rev. C 70064610 (2004).

3. J. M. Carmona, J. Richert and P. Wagner, Eur. Phys. Jour. A 11287 (2001).

4. M. J. Ison and C. O. Dorso, Phys. Rev. C 71064603 (2005).

5. A. Strachan and C. O. Dorso, Phys. Rev. C 55775 (1997).

6. L. G. Moretto, R. Ghetti, L. Phair, K. Tso and G. J. Wozniak, Phys. Rep. 287249 (1997).

7. A. S. Botvina and D. H. E. Gross, Phys. Rev. C 58 R23 (1998). 